\title{
COMPETENCIAS DIGITALES Y HABILIDADES INVESTIGATIVAS EN ESTUDIANTES DE ESTUDIOS GENERALES DE UNA UNIVERSIDAD PRIVADA DE LIMA
}

\section{Digital skills and research abilities in general studies students from a private university of Lima}

\author{
Manuel Encarnación Torres Valladares* \\ Elizabeth Cosi Cruz** \\ Carlos Alberto Peña Miranda***
}

\begin{abstract}
Resumen
El desarrollo del presente trabajo de investigación tiene como propósito fundamental establecer si las competencias digitales se relacionan con las habilidades investigativas en estudiantes de estudios generales de una Universidad Privada de Lima durante el año 2019. Se utilizó un diseño no experimental de nivel correlacional. La muestra de estudio fue probabilística y estuvo compuesta por un total de 348 estudiantes, a quienes se les administró dos instrumentos de evaluación: el cuestionario de competencias digitales de Santiago Mengual y el cuestionario de habilidades investigativas de Jaime Rodríguez. Ambos instrumentos presentan consistentes niveles de validez y confiabilidad. Los resultados indican que las competencias digitales y las habilidades investigativas se encuentran relacionadas significativamente $(r=0,84)$, así como también se encontraron correlaciones significativas entre las diversas competencias digitales y las diversas habilidades investigativas, lo que demuestra la importancia que tienen estas variables en el desarrollo académico de los estudiantes.
\end{abstract}

Palabras clave: Competencias digitales, habilidades investigativas, aprendizaje autónomo, tecnologías de la información, logros de aprendizaje.

\begin{abstract}
The development of this research work has as main objective to establish whether the digital skills are related to the research abilities in students of general studies from a Private University of Lima during the year 2019. A non-experimental correlational research design was used. The studied sample was probabilistic and was composed by a total of 348 students, to whom two evaluation instruments were applied: the Questionnaire of digital skills of Santiago Mengual, and the Questionnaire of research abilities of Jaime Rodriguez. Both instruments have consistent levels of validity and reliability. The results show that the digital skills and the research abilities are significantly correlated $(r=0,84)$. Furthermore, significant correlations between the diverse digital skills and the diverse research abilities were found, which demonstrates the importance these variables have in the academic development of the students.
\end{abstract}

Key words: Digital skills, research abilities, autonomous learning, information technologies, learning achievements.

\footnotetext{
* Doctor. Docente UNMSM. E-mail: manueltorres67@ yahoo.es, Lima - Perú. ORCID 0000-0003-1530-4229

** Doctora. Docente Universidad Norbert Wiener: Elizabeth.cosi@uwiener.edu.pe, Lima - Perú. ORCID 0000-0002-0255-7705

*** Doctor. Docente UNMSM, E-mail: cpenam@unmsm.edu.pe, Lima - Perú. ORCID 0000-0002-4339-4615
}

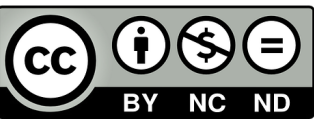

Para utilizar este artículo debe incluir: Autor / Título / ISSN 


\section{INTRODUCCIÓN}

En los últimos años, a raíz del avance extraordinario de la tecnología, las TICs se han vuelto parte de la vida de los seres humanos y de sus instituciones. Así por ejemplo, en el terreno de la Educación es cada vez más notoria la presencia de equipos informáticos en las aulas de clase, se han creado plataformas virtuales y diversidad de técnicas digitales de apoyo al aprendizaje, etc. El objetivo último es formar ciudadanos altamente preparados y capaces de enfrentar los retos que plantea el mundo de hoy.

En este contexto es evidente que la realidad nos exige como sociedad, contar con un proyecto educativo nacional que tenga como objetivo central; desarrollar el conocimiento, que promueva un aprendizaje autónomo, el cual debe permitir la captura o elaboración de nuevas formas de conocimiento. En este proceso el papel del profesor es fundamental, sin embargo, no es mucho lo que se ha avanzado en este aspecto, por lo que es tiempo de que los profesores incursionen decididamente en el mundo de la tecnología del siglo XXI (Colás y Pons, 2004), y para ello requieren desarrollar un conjunto de competencias que se les ha llamado competencias digitales.

Las competencias digitales deben ser entendidas como un conjunto de habilidades y capacidades que va a facilitar el manejo de los dispositivos digitales y sus diferentes aplicaciones, el manejo de las redes para acceder a la información y utilizarlas correctamente. En este camino, resulta importante orientar el uso de las competencias digitales a la búsqueda y creación del conocimiento que permita al estudiante, mejorar sus habilidades de investigación y con ello acrecentar su acervo cognitivo, mejorando significativamente su formación profesional. Todo esto resulta de mayor importancia si se considera que para el año 2020, la ocupación de los puestos de trabajo va a recaer, principalmente, en las personas que posean habilidades digitales.

En este contexto resulta imprescindible desarrollar nuevas metodologías y nuevas estrategias docentes que logren entusiasmar a los estudiantes del siglo XXI. Ellos tienen pleno derecho de ser educados considerando sus necesidades y características. Sin embargo, todavía el docente sigue utilizando tecnología del siglo pasado y le cuesta introducirse en el mundo digital, propio de sus estudiantes. Vivimos una época en la que la trasmisión del conocimiento en un aula de clase ya no es suficiente. Los tiempos actuales demandan el desarrollo de competencias, para lo cual se requiere elaborar una serie de experiencias de aprendizaje que puedan resultar significativas para los estudiantes y en las que, inevitablemente, ellos puedan ser el centro de todo el proceso de enseñanza aprendizaje. En este aspecto, la utilización eficiente de las TICs, es fundamental en tanto se va a colocar al estudiante frente a una realidad que conoce y con la que vive diariamente, (Sánchez, Boix y Jurado, 2009).

El desarrollo de las competencias digitales del profesorado resultan necesarias por variadas razones, principalmente las que están ligadas al propio proceso de evolución de los docentes y las necesidades de satisfacer las exigencias académicas, administrativas y de investigación, que lleven directamente a una mejora sustantiva del aprendizaje y el desarrollo de competencias personales y profesionales de los estudiantes. Por otra parte, debe considerarse que para los estudiantes es un elemento altamente motivador el uso de las TICs en el aula de clases. Esto supone ubicar la educación en un sentido bastante más realista, en tanto, la tecnología ocupa una posición de privilegio.

Este nuevo enfoque sobre el uso de las TICs, tiene una fuerte repercusión, tanto en la manera de entender la educación como en la generación de conocimiento, en la medida que permite acceder rápidamente a la diversidad de investigaciones y bibliotecas virtuales que acercan el conocimiento, permitiendo su desarrollo a partir de la realización de nuevas investigaciones. Desde este punto de vista las TICs están fuertemente vinculadas a los procesos de investigación (Barrón, 2000) y sin duda al desarrollo de habilidades investigativas en los estudiantes.

Bajo estas consideraciones se puede afirmar que la formación en competencias investigativas es una tarea de necesario cumplimiento por parte de las universidades, pues ella permite resolver problemas de la realidad y alcanzar resultados, por lo que su principal característica es su orientación hacia la praxis y la posibilidad de una inserción eficiente en la vida productiva de las personas. Las competencias investigativas deben brindar la posibilidad de articular la diversidad de conocimientos que puedan ir generando los estudiantes, hasta llegar a los conocimientos 
profesionales y las propias experiencias laborales, las que juntas todas ellas deben facilitar la toma de decisiones y la resolución de problemas (Moreno, 2005).

$\mathrm{Al}$ interior de las universidades la generación de nuevos conocimientos no es una actividad restringida sólo a docentes o estudiantes de postgrado, es también una actividad realizada por alumnos de pregrado bajo diversas modalidades. Durante mucho tiempo se ha desestimado la producción de investigación en los estudiantes de pregrado, lo cual no sólo se debe a que los currículos universitarios no ofrecen las competencias necesarias para que los alumnos puedan dar inicio a su formación científica, sino que tampoco se recibe el apoyo económico necesario; el Perú destina sólo el 0,08\% del Producto Bruto Interno a la investigación y desarrollo, mientras que los países desarrollados destinan aproximadamente el 3\% (CONCYTEC, 2016).

Es necesario que la inversión en educación y en investigación se incremente sustantivamente, de lo contrario será muy difícil avanzar hacia el desarrollo que la nación reclama. Por una parte, las universidades van a reducir sus niveles de competitividad, y por otra parte, se seguirán formando profesionales que básicamente serán repetidores y no generadores de conocimientos como ocurre en los países de mayor desarrollo. La investigación científica debe entenderse como un instrumento de desarrollo institucional. La investigación universitaria no debe ser un proceso aislado sino que debe estar integrada y subordinada a las estructuras económicas, política y cultural del país.

Teniendo en cuenta las consideraciones expuestas líneas arriba, la formación de los futuros profesionales, en lo que se refiere a las competencias digitales e investigativas, deviene en una necesidad urgente y en un importante reto que la universidad debe asumir en el corto plazo. La investigación formativa, básica en los procesos de formación profesional, está enfocada al proceso de aprendizaje del estudiante en tanto busca no solo el desarrollo de nuevos conocimientos, sino también como se produce el sistema de interrelación que tienen los seres humanos y como asumen y comparten el conocimiento.

El objetivo principal de la presente investigación está orientado a establecer si las competencias digitales se encuentran relacionadas con las habilidades investigativas en una muestra de estudiantes de estudios generales de una Universidad Privada de Lima. Como consecuencia de lo expuesto, los problemas de la presente investigación se plantean de la siguiente manera:

1. ¿Cuáles son los niveles de competencias digitales que presentan los estudiantes de estudios generales de una Universidad Privada de Lima?

2. ¿Cuáles son los niveles de habilidades investigativas que presentan los estudiantes de estudios generales de una Universidad Privada de Lima?

3. ¿Dequémanera serelacionan las competencias digitales con las habilidades investigativas en estudiantes de estudios generales de una Universidad Privada de Lima?

4. ¿Qué relación existe entre las dimensiones de las competencias digitales y las habilidades investigativas en estudiantes de estudios generales de una Universidad Privada de Lima?

5. ¿Qué relación existe entre las dimensiones de las competencias digitales y las dimensiones de las habilidades investigativas en estudiantes de estudios generales de una Universidad Privada de Lima?

\section{OBJETIVOS}

1. Describir los niveles de competencias digitales que presentan los estudiantes de estudios generales de una Universidad Privada de Lima.

2. Identificar los niveles de habilidades investigativas que presentan los estudiantes de estudios generales de una Universidad Privada de Lima.

3. Establecer la relación que existe entre las competencias digitales y las habilidades investigativas en estudiantes de estudios generales de una Universidad Privada de Lima. 
4. Precisar la relación que existe entre las dimensiones de las competencias digitales y las habilidades investigativas en estudiantes de estudios generales de una Universidad Privada de Lima.

5. Identificar la relación que existe entre las dimensiones de las competencias digitales y las dimensiones de las habilidades investigativas en estudiantes de estudios generales de una Universidad Privada de Lima.

\section{HIPÓTESIS}

1. Las competencias digitales se relacionan significativamente con las habilidades investigativas en estudiantes de estudios generales de una Universidad Privada de Lima.

2. Las dimensiones de las competencias digitales se relacionan significativamente con las habilidades investigativas en estudiantes de estudios generales de una Universidad Privada de Lima.

3. Las dimensiones de las competencias digitales se relacionan significativamente con las dimensiones de las habilidades investigativas en estudiantes de estudios generales de una Universidad Privada de Lima.

\section{MÉTODO}

El método específico que se impone es el método hipotético-deductivo, el mismo que es conceptualizado por Bernal (2010), como "un procedimiento que parte de unas aseveraciones en calidad de hipótesis y busca refutar o falsear tales hipótesis, deduciendo de ellas conclusiones que deben confrontarse con los hechos" (p. 56), tal como se ha hecho a lo largo de la presente investigación, particularmente en lo que se refiere a los resultados alcanzados.

\section{Diseño de investigación}

El diseño que se impone utilizar en el desarrollo del presente trabajo de tesis y que permite probar las hipótesis planteadas, es el diseño no experimental de tipo correlacional transversal, de acuerdo a lo señalado por Hernández, Fernández y Baptista (2014). Este diseño pretende establecer la asociación que puede existir entre dos o más variables en un momento en el tiempo.

Las variables se interrelacionan bajo el siguiente esquema:

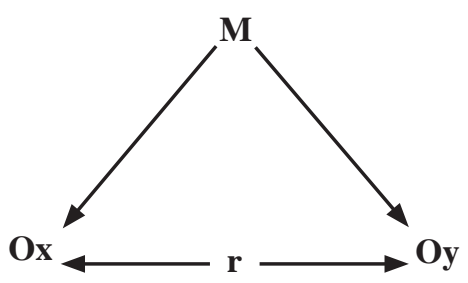

$\mathbf{M}=$ Representa la muestra de estudio

$\mathbf{O x}=$ Representa los datos de la variable competencias digitales

Oy $=$ Representa los datos de la variable habilidades investigativas

$\mathbf{r}=$ Indica el grado de correlación entre ambas variables

\section{Muestra}

La muestra es probabilística de tipo estratificada distribuyendo a los estudiantes considerando la carrera profesional de la cual provienen. La selección de la muestra se realizó utilizando la técnica de muestreo aleatorio y por afijación proporcional. Finalmente, se debe indicar que el tamaño de la muestra se calculó utilizando la fórmula de Sierra Bravo (2003), la misma que dio como resultado que la muestra estuviera compuesta por 348 estudiantes.

\section{Tabla 1}

Composición de la muestra por Ciclo Académico

\begin{tabular}{ccc}
\hline Ciclo & Frecuencia & Porcentaje \\
\hline I & 104 & 29,9 \\
II & 89 & 25,6 \\
III & 68 & 19,5 \\
IV & 87 & 25,0 \\
Total & 348 & 100,0 \\
\hline
\end{tabular}

\section{Instrumentos}

Los instrumentos que se utilizaron en la presente investigación son: el Inventario de Competencias Digitales de Santiago Mengual de la Universidad 
de Alicante y el Cuestionario de Habilidades Investigativas de Jaime Rodríguez de México.

\section{Análisis de validez y confiabilidad de los instrumentos}

Como regularmente ocurre en todo proceso de investigación que se realiza, es necesario que los instrumentos de medición a utilizar cumplan con los requisitos técnicos de validez y confiabilidad. En este proceso es que se debieron realizar los análisis correspondientes que dieron como resultado que, en el caso de la confiabilidad, determinada a partir de la utilización del estadístico alfa de cronbach, el cuestionario de competencias digitales alcanza un valor de 0,96 el mismo que puede ser tipificado como muy bueno. En el caso del cuestionario de habilidades investigativas, el alfa de cronbach alcanzado fue de 0,84 , el cual es igualmente bueno. Para el caso de la validez de los instrumentos, estos se realizaron utilizando el análisis factorial exploratorio. Los resultados encontrados indican que ambas pruebas presentan validez de constructo.

\section{RESULTADOS}

\section{Análisis descriptivos}

Tabla 2.

Test de Bondad de Ajuste a la Curva Normal de Kolmogorov - Smirnov de las Variables estudiadas

\begin{tabular}{lcccc}
\hline Variable & Media & D.E. & K-SZ & Sig. \\
\hline Alfabetización tecnológica & 35,90 & 6,00 &, 069 &, 021 \\
Acceso y uso de la información & 35,06 & 6,01 &, 071 &, 016 \\
Comunicación y colaboración & 35,10 & 5,38 &, 053 &, 200 \\
Ciudadanía digital & 35,87 & 5,48 &, 0749 &, 004 \\
Creatividad e innovación & 34,95 & 6,46 &, 073 &, 012 \\
Habilidades Cognitivas & 16,08 & 2,49 &, 147 &, 000 \\
Habilidades tecnológicas & 17,30 & 2,43 &, 163 &, 000 \\
Habilidades metodológicas & 16,93 & 2,43 &, 141 &, 000 \\
Habilidades para gestionar la investigación & 15,75 & 2,58 &, 095 &, 000 \\
Habilidades para el trabajo en equipo & 19,34 & 3,02 &, 124 &, 000 \\
\hline
\end{tabular}

$\mathrm{N}=348$

El resultado del análisis de normalidad efectuado con el test de bondad de ajuste a la curva normal de Kolmogorov-Smirnov y que se observan en la tabla 2 , muestran que en todas las variables estudiadas, se obtienen coeficientes K-S Z que son estadísticamente significativos, por lo que se puede concluir que no se presentan adecuadas aproximaciones a la curva normal, por lo que es factible utilizar para el análisis de los datos, estadísticos no paramétricas (Siegel y Castellan, 1995). 
Tabla 3

Niveles de Competencias digitales

\begin{tabular}{ccc}
\hline Niveles & Frecuencias & Porcentajes \\
\hline Bajo & 60 & 17,2 \\
Medio & 237 & 68,1 \\
Alto & 51 & 14,7 \\
Total & 348 & 100,0 \\
\hline
\end{tabular}

Los resultados descriptivos que se observan en la tabla 3 , nos informan que las competencias digitales que presentan los estudiantes de la muestra, se encuentran ubicadas en un nivel medio, $68,1 \%$, luego aparecen los estudiantes que se ubican en un nivel bajo con el $17,2 \%$ y finalmente aparecen los que se encuentran en el nivel alto con el $14,7 \%$.

\section{Tabla 4}

Niveles de habilidades investigativas

\begin{tabular}{ccc}
\hline Niveles & Frecuencias & Porcentajes \\
\hline Bajo & 71 & 20,4 \\
Medio & 198 & 56,9 \\
Alto & 79 & 22,7 \\
Total & 348 & 100,0 \\
\hline
\end{tabular}

Los resultados descriptivos que se observan en la tabla 4, nos informan que las habilidades investigativas que presentan los estudiantes de la muestra, se encuentran ubicadas en un nivel medio, $56,9 \%$, luego aparecen los estudiantes que se ubican en un nivel alto con el $22,7 \%$ y finalmente aparecen los que se encuentran en el nivel bajo con el 20,4\%.

\section{Análisis Correlacionales}

\section{Tabla 5}

Análisis de Correlación (rho de Spearman) entre las competencias digitales y las habilidades investigativas

\begin{tabular}{cc}
\hline Variables & Habilidades Investigativas \\
Competencias digitales & $0,84 * * *$ \\
\hline$* \mathrm{p}<.05 * * \mathrm{p}<.01 * * * \mathrm{p}<.001$ & \\
$\mathrm{~N}=348$
\end{tabular}

Los resultados que se muestran en la Tabla 5 , informan que las competencias digitales se correlacionan significativamente con las habilidades investigativas de los estudiantes.

\section{Tabla 6}

Análisis de Correlación (rho de Spearman) entre las diversas áreas de las competencias digitales y las habilidades investigativas

\begin{tabular}{cc}
\hline Variables & Habilidades investigativas \\
\hline Alfabetización tecnológica & $0,76 * * *$ \\
Acceso y uso de la información & $0,71 * * *$ \\
Comunicación y colaboración & $0,75 * * *$ \\
Ciudadanía digital & $0,71 * * *$ \\
Creatividad e innovación & $0,78 * * *$ \\
\hline $\mathrm{p}<.05 * * \mathrm{p}<.01 * * * \mathrm{p}<.001$ & \\
$\mathrm{~N}=348$ &
\end{tabular}

Como se puede observar en la Tabla 6, los resultados nos informan que las diversas competencias digitales se correlacionan significativamente con las habilidades investigativas de los estudiantes.

\section{Tabla 7}

Análisis de las Correlaciones entre las diversas áreas de las competencias digitales y las diversas habilidades investigativas

\begin{tabular}{llllll}
\hline Variables & HCog & HTec & HMet & HGes & HTra \\
\hline Alfabetización tecnológica & $0,63 * *$ & $0,53 * *$ & $0,65 * *$ & $0,59 * *$ & $0,52 * *$ \\
Acceso y uso información & $0,54 * *$ & $0,56 * *$ & $0,58 * *$ & $0,54 * *$ & $0,47 * *$ \\
Comunicación colaboración & $0,57 * *$ & $0,58 * *$ & $0,70 * *$ & $0,57 * *$ & $0,48 * *$ \\
Ciudadanía digital & $0,55 * *$ & $0,52 * *$ & $0,63 * *$ & $0,53 * *$ & $0,46 * *$ \\
Creatividad e innovación & $0,61 * *$ & $0,58^{* *}$ & $0,69 * *$ & $0,56 * *$ & $0,52^{* *}$ \\
\hline
\end{tabular}

$* \mathrm{p}<.05 * * \mathrm{p}<.01 * * * \mathrm{p}<.001$

$\mathrm{N}=348$

HCog = Habilidades Cognitivas, HTec $=$ Habilidades tecnológicas, HMet $=$ Habilidades metodológicas, HGes = Habilidades para gestionar la investigación, HTra = Habilidades para el trabajo en equipo 
Como se puede observar en la Tabla 7, los resultados nos informan que las diversas competencias digitales se correlacionan significativamente con las diversas habilidades investigativas de los estudiantes.

\section{DISCUSIÓN}

Como regularmente debe ocurrir en todo proceso de investigación, en el presente estudio se cumplió rigurosamente con cada una de las exigencias que implica el proceso metodológico a fin de garantizar la validez interna de la investigación. Para ello se tuvo cuidado en acceder adecuadamente a la muestra y aplicar los instrumentos, garantizando su anonimato. Por otro lado, los instrumentos de evaluación que se han utilizado en la presente tesis han debido pasar todos los criterios de validez y confiablidad que se exigen en estos casos. Los análisis de los resultados se efectuaron con los estadísticos que correspondían después de realizado el análisis de normalidad correspondiente.

Los resultados de la investigación indican que, respecto a la primera hipótesis que se formuló; "Las competencias digitales se relacionan significativamente con las habilidades investigativas en estudiantes de estudios generales de una Universidad Privada de Lima", indican que la misma ha sido respaldada, en la medida que el análisis de correlación alcanza un valor de $r=0,84$ el que se puede considerar como alto. Estos resultados refuerzan las propuestas de Sánchez, Boix y Jurado, (2009) y Barrón, (2000), en el sentido de que el manejo de las TICs y el desarrollo de competencias digitales son muy importantes para la formación de los estudiantes, no solo en los procesos de aprendizaje, sino fundamentalmente, en el desarrollo de habilidades investigativas. En esta línea de pensamiento, Vivancos (2008), considera que los estudiantes de esta nueva generación, requieren tener conocimiento sobre aplicaciones informáticas que permitan mejorar significativamente su capacidad de búsqueda y tratamiento de la información, sin perder jamás la actitud crítica, reflexiva, responsable y ética.

Por otra parte, estos resultados concuerdan con las investigaciones realizadas por otros autores como Esteve (2015), Caudillo (2016), Zúñiga (2016) y Pérez (2015), quienes resaltan la necesidad de preparar y capacitar a docentes y estudiantes en el manejo de las nuevas tecnologías y en el desarrollo de competencias digitales, en tanto, deben convertirse en las "capacidades operativas, instrumentales $\mathrm{y}$ actitudinales con las que funcionar y resolver problemas en la era digital y, sin cuyo dominio, los alumnos estarán perdidos y serán analfabetos funcionales", (Pérez, 2015, p. 573).

La importancia manifiesta que tienen las competencias digitales para los estudiantes del siglo XXI, convierte en una necesidad de pronta atención y solución, la incorporación de estas competencias en el currículo universitario en tanto se trata de formar en ellos, los conocimientos, aptitudes, actitudes, habilidades y capacidades indispensables y necesarias que, les permitan adaptarse a una sociedad en constante cambio en todos los ámbitos y eso incluye el conocimiento y la propia educación que tiene ahora, como una nueva obligación, permitirle al estudiante un tránsito saludable hacía la Sociedad del Conocimiento.

Por otra parte, es importante considerar que la existencia de una brecha digital entre los docentes y estudiantes, convierten en necesario y urgente atender las dificultades digitales de los docentes. Si bien es cierto que los estudiantes van al ritmo que marca el desarrollo de la tecnología, los docentes no están en el mismo ritmo, por lo que las diferencias son manifiestas respecto de las competencias digitales lo que no les permite lograr las metas educativas que demandan los tiempos actuales.

\section{CONCLUSIONES}

1. Respecto de las competencias digitales, los resultados de la investigación permiten determinar que las mismas, se encuentran en un nivel medio de forma dominante.

2. Respecto de las habilidades investigativas, los resultados de la investigación permiten determinar que las mismas, se encuentran en un nivel medio de forma dominante.

3. El análisis de las correlaciónales que se realizaron con el apoyo del estadístico rho de Spearman, nos indican la existencia de correlaciones significativas entre las competencias digitales y las habilidades investigativas, en los estudiantes de una Universidad Privada de Lima. 
4. El análisis de las correlaciones que se realizaron con el apoyo del estadístico rho de Spearman, nos indican la existencia de correlaciones significativas entre las diversas competencias digitales y las habilidades investigativas en los estudiantes de una Universidad Privada de Lima.

5. El análisis de las correlaciónales que se realizaron con el apoyo del estadístico rho de Spearman, nos indican la existencia de correlaciones significativas entre las diversas competencias digitales y las diversas habilidades investigativas, en los estudiantes de una Universidad Privada de Lima.

\section{REFERENCIAS}

Barrón, C. (2000). La educación basada en competencias en el marco de los procesos de globalización. En M. A. Valle. Formación en competencias y certificación profesional (pp. 1744). México: Universidad Nacional Autónoma de México.

Bernal, C. (2010). Metodología de la investigación. Pearson editorial. Colombia.

Caudillo, D. (2016). Competencia Digital en el Proceso de Apropiación de las TIC en Jóvenes de Secundaria en el Estado de Sonora, México. Propuesta de Innovación Educativa para la Mejora de las Habilidades Digitales en el Aula. Tesis Doctoral. Universidad de Sonora. México.

Colas, P. y Pons, J. (2004). La formación del profesorado basada en redes de aprendizaje virtual: aplicación de la técnica DAFO. Teoría de la educación: Educación y Cultura en la Sociedad de la Información, núm. 5, pp. 207-222.
Consejo Nacional de Ciencia, Tecnología e Innovación Tecnológica. (2017). I Censo Nacional de Investigación y Desarrollo a Centros de Investigación 2016. Concytec.

Esteve, F. (2015). La competencia digital docente. Análisis de la autopercepción y evaluación del desempeño de los estudiantes universitarios de educación por medio de un entorno 3D. Tesis Doctoral. Universidad Rovira i Virgili. España.

Hernández, R., Fernández, C. y Baptista, P. (2014) Metodología de la Investigación. México. Ed Mc Graw Hill.

Moreno,G(2005).Potenciarlaeducación.Uncurricular transversal de formación para la investigación. Revista Electrónica Iberoamericana sobre calidad, eficacia y cambio en educación. España.

Pérez, A. (2015). Alfabetización Digital y Competencias Digitales en el Marco de la Evaluación Educativa: Estudio en Docentes y Alumnos de Educación Primaria en Castilla y León. Tesis Doctoral. Universidad de Salamanca. España.

Sánchez, A., Boix, J. y Jurado, J. (2009). La sociedad del conocimiento y las TICS: una inmejorable oportunidad para el cambio docente. Píxel Bit, núm. 34, pp. 174-204.

Sierra Bravo, R. (2001). Técnicas de Investigación Social. Teoría y ejercicios. Madrid. Ediciones Paraninfo, S.A.

Vivancos, J. (2008). Tratamiento de la información y competencia digital. Madrid: Alianza Editorial.

Zúñiga, J. (2016). Las competencias digitales en el perfil universitario: El caso de la Facultad de Pedagogía de la Universidad Veracruzana. Tesis Doctoral. Universidad Veracruzana. México.

Fecha de recepción: 21 de noviembre 2019

Fecha de aceptación: 27 de diciembre 2019 